\title{
Peroxisome Proliferator-Activated Receptor Gamma Agonists in Kidney Disease - Future Promise, Present Fears
}

\author{
Zhiguo Mao ${ }^{\mathrm{a}-\mathrm{c}}$ Albert C.M. Ong ${ }^{\mathrm{a}, \mathrm{b}}$ \\ a Kidney Genetics Group, Academic Nephrology Unit, Henry Wellcome Laboratories for Medical Research,

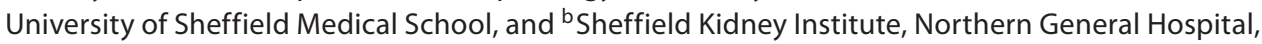 \\ Sheffield Teaching Hospitals Foundation Trust, Sheffield, UK; ' Division of Nephrology, Changzheng Hospital, \\ Second Military Medical University, Shanghai, China
}

\section{Key Words}

PPAR- $\gamma$ agonists $\cdot$ Thiazolidinediones $\cdot$ Kidney disease $\cdot$

Diabetes mellitus

\begin{abstract}
The peroxisome proliferator-activated receptor superfamily (PPARs) comprises a class of nuclear receptors with significant effects in regulating multiple cellular pathways. Much research and clinical interest has surrounded the PPAR- $\gamma$ isoform because of its key role in the transcriptional regulation of metabolic pathways and the efficacy of thiazolidinediones, the most clinically used PPAR- $\gamma$ agonist, in the management of type 2 diabetes mellitus. In this review, we discuss the pathogenic role of PPAR- $\gamma$ in experimental models of kidney disease, clinical trials of thiazolidinediones in diabetic and non-diabetic kidney disease, recent safety concerns surrounding PPAR- $\gamma$ agonists and reflect on their potential use in 'orphan' kidney diseases.
\end{abstract}

Copyright $\odot 2009$ S. Karger AG, Basel
The term peroxisome proliferator-activated receptors (PPARs) arose approximately 18 years ago, when Issemann and Green [1] observed that certain industrial compounds could bind to certain nuclear receptors and cause rodent hepatic peroxisomes to increase in volume and density. Although PPAR- $\gamma$ agonists are not known to induce peroxisome proliferation in humans [2], these unusual nuclear hormone receptors have attracted great attention in recent years as their roles in the transcriptional regulation of key metabolic (including lipid metabolism, adipogenesis and insulin sensitivity), inflammatory, atherosclerotic pathways and in kidney protection have been clarified. As a consequence, thiazolidinediones (TZDs), the most clinically used PPAR- $\gamma$ agonists, have become blockbuster drugs especially in the management of type 2 diabetes mellitus (T2DM). More recently, however, important long-term safety issues have arisen casting doubt over their future clinical use [3].

This review summarizes experimental and clinical evidence for PPAR- $\gamma$ in relation to the kidney disease, recent safety concerns of PPAR- $\gamma$ agonists in clinical use and provides some thoughts on their potential future uses in non-diabetic kidney diseases. 


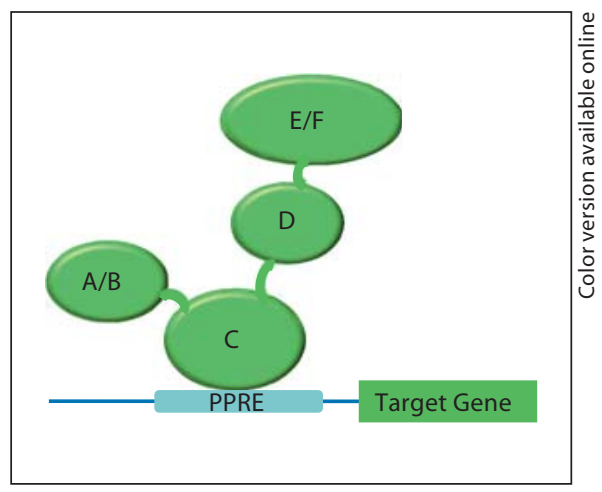

Fig. 1. Structure of PPAR- $\gamma$. A/B $=\mathrm{NH} 2$-terminal ligand-independent transactivation domain; $\mathrm{C}=\mathrm{DNA}$ binding domain; $\mathrm{D}=$ hinge domain; $\mathrm{E} / \mathrm{F}$ = ligand binding domain and ligand-dependent transactivation domain; PPRE $=$ PPAR-responsive element.

\section{The Characteristics of PPAR- $\gamma$ and Its Agonists}

PPAR- $\gamma$ is an isotype of the PPAR superfamily with four major functional domains (fig. 1): an NH2-terminal ligand-independent transactivation domain (A/B), a DNA-binding domain (C), a hinge domain (D) and a $\mathrm{COOH}$-terminal domain including the ligand-binding domain and the ligand-dependent transactivation domain (E/F) [4-6]. To date, two major splice isoforms of PPAR- $\gamma$ (PPAR- $\gamma 1$ and PPAR- $\gamma 2$ ) have been identified in the mouse; two additional human isoforms PPAR- $\gamma 3$ and PPAR- $\gamma 4$ have been found [7].

The highest levels of PPAR- $\gamma$ can be found in adipose tissue with lower levels detected in kidney, urinary bladder, skeletal muscle, liver and heart. In addition, PPAR- $\gamma$ is expressed in the vasculature and various immune cells. In the kidney, PPAR- $\gamma$ is predominantly expressed in the distal medullary collecting ducts but also to a lesser extent in glomeruli, renal microvasculature and proximal tubules. In vitro expression of PPAR- $\gamma$ has been reported in cultured glomerular mesangial cells, podocytes, proximal tubule and collecting duct cells. These findings are the basis of the diverse roles of PPAR- $\gamma$ in regulating renal physiology and pathophysiology.

TZDs, also known as glitazones, were found to improve insulin sensitivity in diabetic animals more than 10 years before PPAR- $\gamma$ was cloned [8]. The activation of PPAR- $\gamma$ by TZD is through a ligand-dependent transactivation mechanism, modulating the transcriptional activity of key target genes. Troglitazone was the first TZD class drug approved for clinical use in 1997 followed by rosiglitazone and pioglitazone in 1999.

PPAR- $\gamma$ Agonists in Kidney Disease

\section{The Beneficial Properties of PPAR- $\gamma$ Agonists in the Kidney}

An enormous body of research has identified beneficial functions of TZDs on the kidney in laboratory and clinical settings, although the exact mechanisms of the renoprotective effects is unclear. The majority of studies have addressed aspects of the metabolic syndrome, i.e. obesity, insulin resistance and dyslipidaemia. As there is evidence correlating metabolic syndrome and the development of chronic kidney disease (CKD), the beneficial effects of TZDs in improving glucose tolerance could indirectly improve CKD progression. More recently, however, investigators have begun to uncover direct effects of TZDs on the kidney such as anti-proteinuric, vascular protective, anti-inflammatory and anti-fibrotic effects which are all independent of the capacity to improve glucose tolerance (fig. 2). TZDs thus have the potential to become attractive therapeutic agents for other kidney diseases apart from diabetic nephropathy (DN).

\section{Reduction in Proteinuria}

To date, most animal models (diabetic and non-DN) and many clinical trials have convincingly demonstrated the anti-proteinuric effect of TZDs $[9,10]$. Since proteinuria (or albuminuria) is a modifiable risk factor for the progression to end-stage renal disease and a marker of endothelial dysfunction, the anti-proteinuric effect of TZDs could be a significant advantage in reducing renal and cardiovascular risk in CKD patients.

\section{Vascular Effects}

Laboratory and clinical data have demonstrated a small but significant effect of TZDs in lowering systemic blood pressure [11] - this could be due to enhanced insulin sensitivity or to a direct action on resistance vessels. Since PPAR- $\gamma$ is expressed in both endothelial and vascular smooth muscle cells, the direct regulation on blood pressure could be through modulating vasoactive factor release by endothelium (endothelin-1, prostacyclin and nitric oxide) or by reducing vascular smooth muscle cells tone (e.g. down-regulating the ANG II type 1 receptor) [12]. A reduction in sympathetic overactivity may also play a role in TZD-mediated BP lowering. As a common side-effect of TZDs is fluid retention, the antihypertensive effect of TZDs could be even more significant in the

Nephron Clin Pract 2009;112:c230-c241 
Fig. 2. Effects of TZDs on kidney.

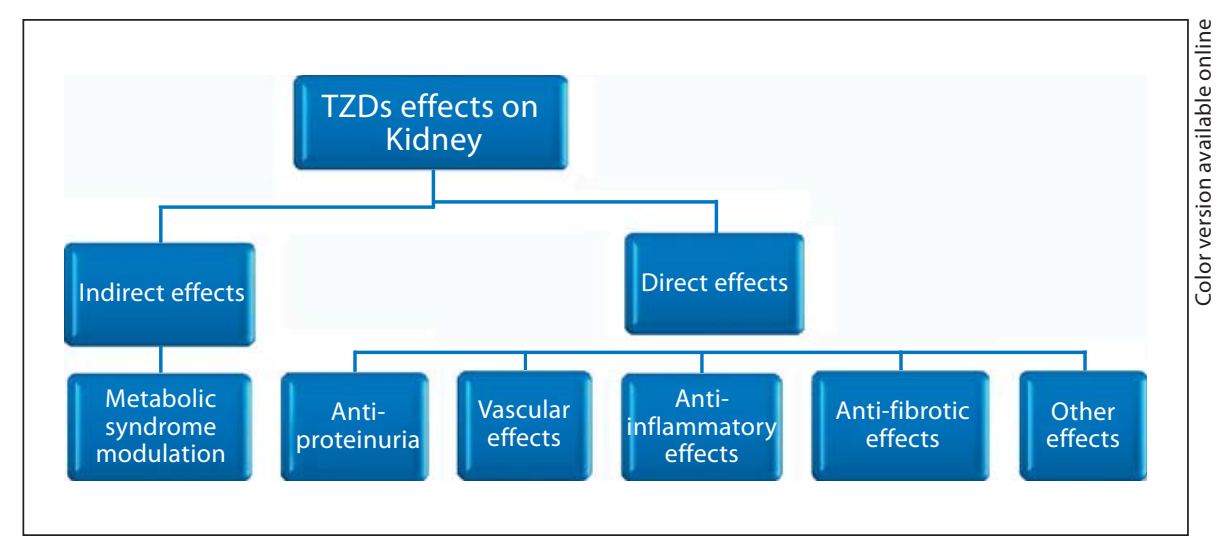

absence of volume expansion. In the kidney, TZDs cause rat post-glomerular efferent arterioles to dilate probably by increasing renal nitric oxide bioavailability and thus can attenuate glomerular hyperfiltration $[13,14]$.

There is evidence of significant cross-talk between PPAR- $\gamma$ signaling and the renin-angiotensin system (RAS). Some angiotensin-receptor blockers (ARB) can activate PPAR- $\gamma$. Conversely, TZDs reduce the production of ANG I and II (A-II) from human subcutaneous adipocytes [15], spontaneously hypertensive rat mesangial cells [16] and down-regulate the A-II type 1 receptor in vascular smooth muscle cells [17]. This has led to other studies showing that TZDs can confer equal or superior renal protection to RAS inhibitors, making the combination of both compounds an attractive therapeutic option $[18,19]$.

\section{Anti-Inflammatory Effects}

TZDs have prominent local and systemic anti-inflammatory effects. They can suppress cytokine synthesis by a number of cell types such as macrophages, renal mesangial cells and renal tubular cells. The range of cytokines studied includes interleukin (IL)-1, IL-6, macrophage chemoattractant protein-1, intercellular adhesion molecule-1 (ICAM-1), vascular cell adhesion molecule-1 and tumor necrosis factor- $\alpha$. In patients with overt $\mathrm{DN}$, TZDs has been shown to reduce markers of systemic inflammation such as plasma IL-6 and C-reactive protein [20, 21].

Pioglitazone attenuates disease progression in a rat model of T2DM by down-regulating NF- $\mathrm{B}$, transforming growth factor (TGF)- $\beta 1$, plasminogen activator inhibitor type-1 (PAI-1) and VEGF [22]. Other studies showed that rosiglitazone attenuates pro-inflammatory responses (increased IL-6, A-II and TGF- $\beta$ production) and stimulates anti-inflammatory responses (through IL-4 synthesis and restoring nitric oxide availability) in the remnant kidney [23] and ureteric obstruction models [24]. TZDs could also directly attenuate the renal injury caused by free radicals and oxidant stress, which themselves lead to inflammation $[25,26]$.

\section{Anti-Fibrotic Effects}

Sclerosis and fibrosis are common end points for many chronic and severe renal diseases: TGF- $\beta$ is believed to play a crucial role in this process. TZDs could reduce kidney fibrosis and sclerosis by attenuating the hyperinsulinaemia and hyperglycaemia which are important stimulators for the production of TGF- $\beta$ from renal mesangial cells and proximal tubule epithelial cells [27-29]. In addition, TZDs have been shown to decrease glomerular TGF- $\beta$ directly independent of serum insulin or glucose $[18,30-34]$, or indirectly by inhibiting A-II synthesis. TZDs could increase matrix degradation by modulating matrix metalloproteinases and PAI-1 secretion $[35,36]$. A direct inhibitory effect of pioglitazone on human renal fibroblast proliferation has been demonstrated [37].

\section{Other Effects}

\section{Acute Kidney Injury}

TZDs reduce ICAM-1 expression and PMN infiltration following ischaemia-reperfusion injury [38-40]. Pioglitazone additionally can reduce cyclosporine nephrotoxicity in allografts $[41,42]$. With the high incidence of 
post-transplantation diabetes mellitus, the use of TZDs in post-transplant management may confer additional benefit over other oral diabetic agents.

\section{Polycystic Kidney Disease}

Of interest, maternal administration of pioglitazone improved survival of Pkd1null embryos, ameliorated the cardiac defects and reduced renal cystic enlargement. Since patients with autosomal dominant polycystic kidney disease account for $10 \%$ of all end-stage renal disease (ESRD) cases, further confirmatory and clinical studies are needed to test the potential use of TZDs in PKD disease progression [43].

\section{PPAR- $\gamma$ Gene Polymorphisms}

In recent years, numerous studies have investigated the possible role of genetic polymorphisms of PPAR- $\gamma$ as susceptibility factors. The C1431T polymorphism was reported to be associated with higher plasma leptin levels and obesity $[44,45]$. The Prol2Ala polymorphism has been linked to a higher incidence of DN in T2DM [46], accelerated GFR loss in T1DM nephropathy, higher ESRD and all-cause mortality in T1DM nephropathy [47]. Although the significance of PPAR- $\gamma$ polymorphisms in different ethnic populations could vary [48], these observations have contributed to heightened interest in the pathological role of PPAR- $\gamma$ in kidney disease, especially in $\mathrm{DN}$.

\section{Clinical Application of TZDs}

\section{Diabetic Nephropathy}

In the past decade, a large number of clinical trials have demonstrated that TZDs reduce urinary albumin excretion, lower blood pressure and suppress systemic inflammation in normotensive or hypertensive patients with DN. Schernthaner et al. [49] reported a 12-month parallel-group double-blind trial comparing pioglitazone and metformin in T2DM patients. This trial recruited 1,199 patients from 167 centers in 12 countries in Europe. The urinary albumin/creatinine ratio was reduced by $19 \%$ in the pioglitazone group but remained unchanged in the metformin group. The incidence of overall and cardiovascular adverse events was similar between both groups. Similarly, a number of other investigators compared TZDs (troglitazone, pioglitazone and rosiglitazone) with placebo or traditional diabetic medication (metfor- min, glibenclamide, insulin etc.) in T2DM patients in different clinical trials and found a consistent efficacy of TZDs on lowering urinary protein excretion and blood pressure [50-56]. More recently, the effects of pioglitazone and glipizide on oxidative stress and inflammation in patients with advanced DN were compared in a randomized, open-label, blinded end-point, 16-week trial [21]. Of interest, pioglitazone reduced the WBC count, C-reactive protein, IL-6 and MMP-9 indicating an antiinflammatory effect in patients with overt DN. Miyazaki et al. [20] compared the effect of rosiglitazone with placebo in T2DM patients and found that urinary albuminto-creatinine ratio was significantly decreased by rosiglitazone after 3 months' treatment. Jin and Pan [57] investigated the effects of a pioglitazone and losartan combination versus losartan monotherapy on CKD progression in $60 \mathrm{~T} 2 \mathrm{DM}$ patients over a 12-month study period. They found that combination therapy significantly slowed GFR decline compared to the losartan-only group.

\section{Other Kidney Diseases}

To date, only one clinical trial examining the therapeutic effects of TZDs in non-T2DM patients has been published. In this open-label randomized crossover study comparing rosiglitazone and RAS blockers (ARB, AECI or both), the subjects enrolled included IgA nephropathy (17/39), focal and segmental glomerulosclerosis (6/39), obesity-related focal and segmental glomerulosclerosis (6/39), reflux nephropathy and other kidney diseases. With rosiglitazone treatment, there was a decrease in urinary protein and systolic blood pressure [58]. However, it should be noted that $78 \%$ of the participants were overweight/obese and $\sim 8 \%$ subjects also had diabetes. The beneficial effect observed could relate to the metabolic improvement seen with TZDs rather than to a direct effect on the kidney.

A phase I study of rosiglitazone $\left(3 \mathrm{mg} / \mathrm{m}^{2} /\right.$ day for 16 weeks) in young patients with primary focal segmental glomerulosclerosis (FSGS) suggested it was safe and welltolerated, although oral clearance was increased by up to $300 \%$ [59]. It seems likely that phase II/III trials will be conducted in FSGS patients.

It should be noted that all the trials performed so far (with one exception) have studied DN patients and relied on surrogate markers of disease progression rather than harder clinical end points such as ESRD or GFR decline. The latter are needed to establish the benefit-risk ratio of using TZDs in both DN and non-DN patients especially in the light of more recent safety concerns regarding long- 
term TZD use (see below). Nevertheless, there is an impressive body of pre-clinical (table 1) and clinical (table 2) data which support further exploration of TZD use in other kidney diseases.

\section{Concerns on the Safety of TZDs}

One advantage of TZDs is that they do not require dose reduction in patients with impaired renal function and can thus be readily used in advanced kidney disease and kidney transplantation patients $[26,60]$. TZDs are generally well tolerated, but their administration has been associated with several prominent side-effects and a number of safety issues. Troglitazone, the first TZD approved for clinical use, was withdrawn in 1999 due to hepatotoxicity. More recently, a meta-analysis of clinical trials on TZDs has raised concerns over potential cardiotoxicity [61].

\section{Hepatotoxicity}

Hepatotoxicity is one of the most studied complications in the use of TZDs because of several cases of severe liver failure reported with troglitazone, but is rare with rosiglitazone and pioglitazone. Conversely, recent studies suggest that these TZDs may protect against certain forms of liver injury such as chemical-induced liver toxicity and non-alcoholic steatohepatitis [62, 63]. Indeed, some have suggested chronic TZD treatment could help to treat non-alcoholic steatohepatitis [64]. Studies on liver cell-specific PPAR- $\gamma$-null mice could help to clarify the liver-specific roles of PPAR- $\gamma$.

\section{Other Organs}

In vitro reports that TZDs are nephrotoxic $[65,66]$ and carcinogenic [67] have not been confirmed.

\section{Fluid Retention}

Fluid retention has long been recognized as a common side effect of TZDs. It could be due to up-regulation of the epithelial sodium channel $[68,69]$, increased sympathetic nervous system activity or altered endothelial permeability [70, 71]. Fluid retention accounts for at least $75 \%$ of body weight gain in patients receiving TZDs $[72,73]$, and fur- thermore contributes to the increased incidence of congestive heart failure (see below). Whether diuretics can effectively reverse the fluid overload is still controversial [74].

\section{Cardiovascular System}

\section{Increased Heart Failure}

The PROactive study was the first to raise alarm over the potential cardiac side effects of TZDs. This investigated the effects of pioglitazone versus non-TZD antidiabetic therapy on a combined vascular end point in T2DM patients with known vascular disease. They found that patients treated with pioglitazone had a higher incidence of congestive heart failure (CHF) [75].

\section{Cardiovascular Mortality and TZDs}

In 2007, Nissen and Wolski [61] published an influential meta-analysis of all randomized controlled TZD trials in T2DM patients. Compared with the control group, rosiglitazone was associated with a significant increase in the risk of myocardial infarction (odds ratio 1.43) and a borderline significant increase in the risk of death from cardiovascular causes (odds ratio 1.64). As a consequence, the US Food and Drug Administration added a 'black box' warning on the label of TZDs of the increased risks of congestive heart failure.

Since this report, not all subsequent studies have confirmed this gloomy prognosis. TZDs do not adversely affect myocytes or myocardial function [76]. Conversely, emerging data suggest that TZDs may even be cardioprotective following acute ischaemic injury [77-79]. It is notable that in the PROactive study, T2DM patients receiving pioglitazone did not have a significant difference in CHF-related mortality compared to placebo [75]. The meta-analysis by Lago et al. [80] showed that TZDs increased the risk for CHF in patients with T2DM and prediabetes but did not increase the risk of cardiovascular death, results similar to the PROactive study. An interim analysis from the ongoing RECORD study, a long-term multicentre randomized open-label study to evaluate cardiovascular outcomes in patients with T2DM treated with rosiglitazone published following Nissen and Wolski's [61] meta-analysis, did not demonstrate an excess risk of death in rosiglitazone compared to comparator drug groups. However, these authors did find that rosiglitazone was associated with an increased risk of CHF [81]. Finally, a recent meta-analysis re-analysed the same data set as Nissen and Wolski [61] using different modeling and weighting methods, but concluded that neither in- 
Table 1. TZDs in experimental models in kidney disease

\begin{tabular}{|c|c|c|c|c|c|}
\hline Authors, year ${ }^{1}$ & TZD type & Animal model & Effect on urine and BP & $\begin{array}{l}\text { Effect on renal } \\
\text { morphology/function }\end{array}$ & Possible mechanism \\
\hline $\begin{array}{l}\text { Yoshioka et al. } \\
1993[1]\end{array}$ & troglitazone & obese Zucker rats & $\begin{array}{l}\text { urine protein excretion } \\
\text { decrease; BP decrease }\end{array}$ & NA & improve insulin resistance \\
\hline $\begin{array}{l}\text { Buchanan et al. } \\
1995[2]\end{array}$ & pioglitazone & $\begin{array}{l}\text { fructose-fed and } \\
\text { chow-fed rats }\end{array}$ & BP decrease & NA & $\begin{array}{l}\text { inhibit agonist-mediated } \\
\text { calcium uptake by VSMC }\end{array}$ \\
\hline $\begin{array}{l}\text { Fujii et al. } \\
1997[3]\end{array}$ & troglitazone & $\begin{array}{l}\text { streptozotocin-induced } \\
\text { diabetic rats }\end{array}$ & $\begin{array}{l}\text { urine albumin excretion } \\
\text { decrease; BP unchanged }\end{array}$ & NA & NA \\
\hline $\begin{array}{l}\text { Yoshimoto et al. } \\
1997[4]\end{array}$ & pioglitazone & diabetic Wistar fatty rats & $\begin{array}{l}\text { proteinuria decrease; } \\
\text { BP decrease }\end{array}$ & $\begin{array}{l}\text { prevent glomerulosclerosis } \\
\text { and renal arteriolosclerosis }\end{array}$ & NA \\
\hline $\begin{array}{l}\text { Uchida et al. } \\
1997[5]\end{array}$ & pioglitazone & sucrose-fed SHR rats & $\begin{array}{l}\text { attenuate the develop- } \\
\text { ment of hypertension }\end{array}$ & NA & $\begin{array}{l}\text { improve insulin resistance } \\
\text { and hyperinsulinaemia }\end{array}$ \\
\hline $\begin{array}{l}\text { Buckingham et al. } \\
1998 \text { [6] }\end{array}$ & rosiglitazone & obese Zucker rats & $\begin{array}{l}\text { proteinuria decrease; } \\
\text { BP decrease }\end{array}$ & $\begin{array}{l}\text { prevent glomerulosclerosis } \\
\text { and tubulointerstitial fibrosis }\end{array}$ & NA \\
\hline $\begin{array}{l}\text { Fujiwara et al. } \\
2000[7]\end{array}$ & troglitazone & $\begin{array}{l}\text { heminephrectomized } \\
\text { Wistar fatty rats }\end{array}$ & $\begin{array}{l}\text { proteinuria decrease; } \\
\text { BP decrease }\end{array}$ & NA & improve insulin sensitivity \\
\hline $\begin{array}{l}\text { Isshiki et al. } \\
2000[8]\end{array}$ & troglitazone & $\begin{array}{l}\text { streptozotocin-induced } \\
\text { diabetic rats }\end{array}$ & albuminuria decrease & NA & $\begin{array}{l}\text { inhibit the DAG-PKC-ERK } \\
\text { pathway }\end{array}$ \\
\hline $\begin{array}{l}\text { Nicholas et al. } \\
2001[9]\end{array}$ & troglitazone & $\begin{array}{l}\text { streptozotocin-induced } \\
\text { diabetic rats }\end{array}$ & $\begin{array}{l}\text { albuminuria decrease; } \\
\text { BP unchanged }\end{array}$ & NA & $\begin{array}{l}\text { inhibit mesangial growth or } \\
\text { PAI-1 expression }\end{array}$ \\
\hline $\begin{array}{l}\text { Yoshida et al. } \\
2001[10]\end{array}$ & troglitazone & 5/6 nephrectomized SHR & $\begin{array}{l}\text { albuminuria unchanged; } \\
\text { BP decrease }\end{array}$ & $\begin{array}{l}\text { decrease serum creatinine and } \\
\text { glomerular sclerosis indices }\end{array}$ & NA \\
\hline $\begin{array}{l}\text { Ma et al. } \\
2001[11]\end{array}$ & troglitazone & $\begin{array}{l}5 / 6 \text { nephrectomized } \\
\text { SD rats }\end{array}$ & $\begin{array}{l}\text { albuminuria decrease; } \\
\text { BP unchanged }\end{array}$ & prevent glomerulosclerosis & $\begin{array}{l}\text { regulate glomerular cell } \\
\text { proliferation and decreasing } \\
\text { PAI- } 1, \text { TGF- } \beta \text { expression }\end{array}$ \\
\hline $\begin{array}{l}\text { Yamashita et al. } \\
2002[12]\end{array}$ & $\begin{array}{l}\text { troglitazone, } \\
\text { pioglitazone }\end{array}$ & $\begin{array}{l}\text { streptozotocin-induced } \\
\text { diabetic SHR }\end{array}$ & $\begin{array}{l}\text { urine albumin excretion } \\
\text { decrease; } \mathrm{BP} \text { unchanged }\end{array}$ & $\begin{array}{l}\text { prevent anionic sites loss of } \\
\text { glomerular basement } \\
\text { membranes; CCr unchanged }\end{array}$ & NA \\
\hline $\begin{array}{l}\text { Diep et al. } \\
2002[13]\end{array}$ & $\begin{array}{l}\text { troglitazone, } \\
\text { pioglitazone }\end{array}$ & $\begin{array}{l}\text { A-II-induced hypertensive } \\
\text { SD rats }\end{array}$ & $\begin{array}{l}\text { attenuate development } \\
\text { of hypertension }\end{array}$ & NA & NA \\
\hline $\begin{array}{l}\text { Baylis et al. } \\
2003[14]\end{array}$ & rosiglitazone & obese Zucker rats & $\begin{array}{l}\text { proteinuria decrease; } \\
\text { BP unchanged }\end{array}$ & $\begin{array}{l}\text { prevent renal fibrosis, superior } \\
\text { to ACEI; additive protection on } \\
\text { GFR and tubulointerstitial dam- } \\
\text { age when combined with ACEI }\end{array}$ & NA \\
\hline $\begin{array}{l}\text { Tanimoto et al. } \\
2004[15]\end{array}$ & pioglitazone & diabetic KK/Ta mice & $\begin{array}{l}\text { albuminuria decrease; } \\
\text { BP unchanged }\end{array}$ & $\begin{array}{l}\text { prevent glomerular enlarge- } \\
\text { ment in early diabetic disease }\end{array}$ & $\begin{array}{l}\text { improve glomerular } \\
\text { hyperfiltration }\end{array}$ \\
\hline $\begin{array}{l}\text { Dobrian et al. } \\
2004[16]\end{array}$ & pioglitazone & $\begin{array}{l}\text { obese, hypertensive } \\
\text { SD rats }\end{array}$ & prevent hypertension & NA & $\begin{array}{l}\text { reduce free radical } \\
\text { production and increase } \\
\text { nitric oxide availability }\end{array}$ \\
\hline $\begin{array}{l}\text { Khan et al. } \\
2005 \text { [17] }\end{array}$ & rosiglitazone & obese Zucker rats & $\begin{array}{l}\text { albuminuria decrease; } \\
\text { BP decrease }\end{array}$ & NA & NA \\
\hline $\begin{array}{l}\text { Yang et al. } \\
2006[18]\end{array}$ & pioglitazone & $\begin{array}{l}\text { puromycin amino- } \\
\text { nucleoside nephropathy } \\
\text { SD rats }\end{array}$ & $\begin{array}{l}\text { proteinuria and BP } \\
\text { unchanged }\end{array}$ & $\begin{array}{l}\text { improve progression of } \\
\text { glomerulosclerosis }\end{array}$ & $\begin{array}{l}\text { reduce infiltration } \\
\text { glomerular macrophages } \\
\text { and PAI-1 expression }\end{array}$ \\
\hline $\begin{array}{l}\text { Benigni et al. } \\
2006 \text { [19] }\end{array}$ & $\begin{array}{l}\text { pioglitazone } \\
\text { vs. ARB }\end{array}$ & $\begin{array}{l}\text { passive Heymann nephritis } \\
\text { rats }\end{array}$ & $\begin{array}{l}\text { reduced proteinuria as } \\
\text { candesartan }\end{array}$ & $\begin{array}{l}\text { ameliorate functional and } \\
\text { structural renal damage }\end{array}$ & $\begin{array}{l}\text { enhance nephrin gene } \\
\text { transcription }\end{array}$ \\
\hline $\begin{array}{l}\text { Ohga et al. } \\
2007[20]\end{array}$ & pioglitazone & STZ-inducible DM rats & albuminuria decrease & reduce glomerular hypertrophy & anti-inflammatory action \\
\hline $\begin{array}{l}\text { Ohtomo et al. } \\
2007[21]\end{array}$ & pioglitazone & $\begin{array}{l}\text { spontaneously hyper- } \\
\text { tensive/NIH-corpulent } \\
\text { rat (SHR/NDmcr-cp) }\end{array}$ & proteinuria decrease & $\begin{array}{l}\text { improve renal function; } \\
\text { reduce glomerular and } \\
\text { tubulointerstitial fibrosis }\end{array}$ & $\begin{array}{l}\text { reduce intrarenal TGF- } \beta \\
\text { expression; normalize } \\
\text { insulin levels }\end{array}$ \\
\hline
\end{tabular}


Table 1 (continued)

\begin{tabular}{|c|c|c|c|c|c|}
\hline Authors, year ${ }^{1}$ & TZD type & Animal model & Effect on urine and BP & $\begin{array}{l}\text { Effect on renal } \\
\text { morphology/function }\end{array}$ & Possible mechanism \\
\hline $\begin{array}{l}\text { Doi et al. } \\
2007[22]\end{array}$ & Rosiglitazone & $\begin{array}{l}\text { renal ischemia-reperfusion } \\
\text { injury rat }\end{array}$ & NA & decrease in apoptosis & induce HGF expression \\
\hline $\begin{array}{l}\text { Omasu et al. } \\
2007[23]\end{array}$ & pioglitazone & $\begin{array}{l}\text { spontaneously } \\
\text { hypercholesterolemic rats }\end{array}$ & proteinuria decrease & $\begin{array}{l}\text { preserve renal function; reduce } \\
\text { renal fibrosis as candesartan }\end{array}$ & inhibit PAI-1 \\
\hline $\begin{array}{l}\text { Namikoshi et al. } \\
2007[24]\end{array}$ & pioglitazone & $\begin{array}{l}\text { obese Zucker rats fed high } \\
\text { protein diet }\end{array}$ & $\begin{array}{l}\text { proteinuria and } \\
\text { BP decrease }\end{array}$ & $\begin{array}{l}\text { improve glomerular and } \\
\text { interstitial fibrosis }\end{array}$ & reduce nitrative stress \\
\hline $\begin{array}{l}\text { Westerweel et al. } \\
2008[25]\end{array}$ & rosiglitazone & $\begin{array}{l}\text { anti-Thy1-glomerulo- } \\
\text { nephritis rats }\end{array}$ & proteinuria decrease & preserve renal function & $\begin{array}{l}\text { reduce renal cortical } \\
\text { TGF- } \beta \text {, PAI-1, and } \\
\text { fibronectin-1 expression }\end{array}$ \\
\hline $\begin{array}{l}\text { Ko et al. } \\
2008[26]\end{array}$ & pioglitazone & $\begin{array}{l}\text { Otsuka Long-Evans } \\
\text { Tokushima Fatty rats }\end{array}$ & $\begin{array}{l}\text { albuminuria decrease; } \\
\text { BP unchanged }\end{array}$ & ameliorate glomerulosclerosis & $\begin{array}{l}\text { attenuate renal } \\
\text { inflammatory process }\end{array}$ \\
\hline $\begin{array}{l}\text { Namikoshi et al. } \\
2008 \text { [27] }\end{array}$ & $\begin{array}{l}\text { pioglitazone } \\
\text { combined } \\
\text { with ARB }\end{array}$ & $\begin{array}{l}\text { obese Zucker rats fed high } \\
\text { protein diet }\end{array}$ & $\begin{array}{l}\text { augment antihypertensive } \\
\text { and anti-proteinuric } \\
\text { effect of ARB }\end{array}$ & $\begin{array}{l}\text { further improvement in renal } \\
\text { interstitial fibrosis over ARB } \\
\text { alone }\end{array}$ & NA \\
\hline $\begin{array}{l}\text { Sen et al. } \\
2008[28]\end{array}$ & ciglitazone & $\begin{array}{l}\text { alloxan-inducible DM } \\
\text { C57BL/6J mice }\end{array}$ & NA & $\begin{array}{l}\text { normalize hyperfiltration } \\
\text { induced by DM }\end{array}$ & $\begin{array}{l}\text { decrease glomerular } \\
\text { homocysteine content }\end{array}$ \\
\hline $\begin{array}{l}\text { Kawai et al. } \\
2009 \text { [29] }\end{array}$ & troglitazone & UUO C57BL/6J mice & NA & $\begin{array}{l}\text { reduce renal interstitial } \\
\text { fibrosis and inflammation }\end{array}$ & reduce TGF- $\beta$ expression \\
\hline
\end{tabular}

$\mathrm{NA}=$ Not available; $\mathrm{BP}=$ blood pressure; $\mathrm{VSMC}=$ vascular smooth muscle cells .

${ }^{1}$ The complete reference list for this table is available as online supplementary material (www.karger.com/doi/10.1159/000224789).

creased nor decreased risk could be established for myocardial infarction and cardiovascular death in T2DM patients taking rosiglitazone [82].

Most of these studies have been conducted on patients with normal or near-normal kidney function. However, a recent post hoc analysis of the PROactive study data found that pioglitazone treatment decreased the tendency for CKD patients (eGFR $<60 \mathrm{ml} / \mathrm{min} / 1.73 \mathrm{~m}^{2}$ ) to reach a composite end point of all-cause death, myocardial infarction, and stroke independent of the severity of renal impairment [83]. Although promising, these data require direct confirmation in an RCT of a CKD patient population.

\section{TZDs and Serum Lipids}

The reported effects of rosiglitazone and pioglitazone on lipid profiles have been inconsistent. In 2004, a metaanalysis of 23 RCTs in T2DM patients concluded that both TZDs raised HDL-cholesterol but had different effects on triglyceride and LDL-cholesterol levels [84]. However, more recent studies have found that both drugs raise LDL-cholesterol and LDL particle size in T2DM patients [85]. It is unclear what effects TZDs have on serum lipids in non-T2DM patients. Given these opposite effects, it seems unlikely that there is a class effect on car- diovascular mortality mediated through alterations in circulating lipids.

\section{Clinical Guidelines for TZD Use}

The patient's volume status may be the most important factor to consider prior to starting TZDs. A recent consensus statement from the American Heart Association and American Diabetes Association recommended that TZDs should not be used in patients with NYHA class III or IV for obvious reasons. In patients without established heart disease but with risk factors for congestive heart failure, TZDs should be started at low doses and gradually increased with close monitoring for signs of fluid retention and heart failure [86].

\section{Summary}

Since their initial identification, the PPAR- $\gamma$ nuclear hormone receptor and its major agonist TZDs have rapidly emerged as key regulators of metabolic and other disease pathways. The recognition that TZDs regulate the 
Table 2. Clinical trials of TZDs in kidney patients

\begin{tabular}{|c|c|c|c|c|c|c|c|c|}
\hline Type of patients & Authors, year ${ }^{1}$ & Study design & $\begin{array}{l}\mathrm{Pa}- \\
\text { tients } \\
\mathrm{n}\end{array}$ & Regimens & Duration & $\begin{array}{l}\text { Effects on urine } \\
\text { protein vs. } \\
\text { control/baseline }\end{array}$ & $\begin{array}{l}\text { Effects on } \\
\mathrm{BP}, \mathrm{mm} \mathrm{Hg}\end{array}$ & $\begin{array}{l}\text { Effects on renal } \\
\text { function or other } \\
\text { important factors }\end{array}$ \\
\hline T2DM & $\begin{array}{l}\text { Sironi et al. } \\
1997[30]\end{array}$ & $\begin{array}{l}\text { randomized } \\
\text { controlled }\end{array}$ & 40 & $\begin{array}{l}200 \mathrm{mg} \text { Tro vs. } \\
\text { placebo }\end{array}$ & 8 weeks & unchanged & unchanged & NA \\
\hline T2DM with MA & $\begin{array}{l}\text { Imano et al. } \\
1998[31]\end{array}$ & $\begin{array}{l}\text { randomized } \\
\text { controlled }\end{array}$ & 30 & 400 mg Tro vs. Met & 12 weeks & $-38.5 \%$ & unchanged & NA \\
\hline T2DM with MA & $\begin{array}{l}\text { Nakamura et al. } \\
2000[32]\end{array}$ & $\begin{array}{l}\text { randomized } \\
\text { controlled }\end{array}$ & 45 & $\begin{array}{l}30 \mathrm{mg} \text { Pio vs. } 5 \mathrm{mg} \\
\text { Gli vs. } 0.6 \mathrm{mg} \text { Vog }\end{array}$ & 3 months & $-66 \%$ & unchanged & NA \\
\hline T2DM & $\begin{array}{l}\text { Lebovitz et al. } \\
2001[33]\end{array}$ & $\begin{array}{l}\text { randomized } \\
\text { controlled }\end{array}$ & 493 & $\begin{array}{l}4 \text { or } 8 \mathrm{mg} \text { Ros vs. } \\
\text { placebo }\end{array}$ & 26 weeks & $\begin{array}{l}-22 \% \text { with } \\
8 \mathrm{mg}\end{array}$ & NA & NA \\
\hline $\begin{array}{l}\text { T2DM with MA or } \\
\text { macroalbuminuria }\end{array}$ & $\begin{array}{l}\text { Nakamura et al. } \\
2001[34]\end{array}$ & $\begin{array}{l}\text { randomized } \\
\text { controlled }\end{array}$ & 32 & $\begin{array}{l}400 \text { mg Tro vs. } \\
5 \text { mg Gli }\end{array}$ & 12 months & $-67 \%$ with MA & unchanged & NA \\
\hline $\begin{array}{l}\text { T2DM with MA, } \\
\text { normotensive }\end{array}$ & $\begin{array}{l}\text { Nakamura et al. } \\
2001[35]\end{array}$ & controlled & 28 & $\begin{array}{l}30 \text { mg Pio vs. } \\
\text { placebo }\end{array}$ & 6 months & $-58.9 \%$ & unchanged & NA \\
\hline T2DM & $\begin{array}{l}\text { Bakris et al. } \\
2003[36]\end{array}$ & $\begin{array}{l}\text { open-label, } \\
\text { randomized }\end{array}$ & 129 & 8 mg Ros vs. Gli & 1 year & $-30 \%$ & $-0.1 /-2.3$ & NA \\
\hline T2DM & $\begin{array}{l}\text { Aljabri et al. } \\
2004 \text { [37] }\end{array}$ & $\begin{array}{l}\text { open-label, random- } \\
\text { ized controlled }\end{array}$ & 62 & $\begin{array}{l}\text { 30-45 mg Pio vs. } \\
\text { insulin }\end{array}$ & 16 weeks & $\begin{array}{l}\text { similar to } \\
\text { control }\end{array}$ & $\begin{array}{l}\text { similar to } \\
\text { control }\end{array}$ & NA \\
\hline T2DM with MA & $\begin{array}{l}\text { Yanagawa et al. } \\
2004[38]\end{array}$ & controlled & 40 & Pio vs. Gli & 12 weeks & $\begin{array}{l}\text { similar to } \\
\text { control }\end{array}$ & NA & NA \\
\hline T2DM & $\begin{array}{l}\text { Hanefeld et al. } \\
2004 \text { [39] }\end{array}$ & $\begin{array}{l}\text { multicentre, random- } \\
\text { ized double-blind }\end{array}$ & 639 & $\begin{array}{l}15-45 \mathrm{mg} \text { Pio vs. } \\
850-2,550 \mathrm{mg} \text { Met }\end{array}$ & 1 year & $-15 \%$ & NA & NA \\
\hline T2DM & $\begin{array}{l}\text { Schernthaner } \\
\text { et al., } 2004 \text { [40] }\end{array}$ & $\begin{array}{l}\text { multicenter, random- } \\
\text { ized double-blind }\end{array}$ & 1,199 & $\begin{array}{l}15-45 \text { mg Pio vs. } \\
850-2,550 \text { mg Met }\end{array}$ & 1 year & $-19 \%$ & NA & NA \\
\hline T2DM & $\begin{array}{l}\text { Matthews et al. } \\
2005[41]\end{array}$ & $\begin{array}{l}\text { randomized double- } \\
\text { blind }\end{array}$ & 630 & $\begin{array}{l}\text { 15-45 mg Pio vs. } \\
80-320 \text { mg Gli }\end{array}$ & 1 year & $-10 \%$ & NA & NA \\
\hline Overt DN & $\begin{array}{l}\text { Agarwal et al. } \\
2005[42]\end{array}$ & $\begin{array}{l}\text { open-label, blinded } \\
\text { randomized end point } \\
\text { trial }\end{array}$ & 44 & Pio vs. Gli & 4 months & unchanged & unchanged & NA \\
\hline $\begin{array}{l}\text { T2DM } \\
\text { with/without MA }\end{array}$ & $\begin{array}{l}\text { Pistrosch et al. } \\
2005[43]\end{array}$ & $\begin{array}{l}\text { double-blind } \\
\text { crossover trial }\end{array}$ & 19 & $\begin{array}{l}\text { with MA: Ros vs. } \\
\text { placebo; without } \\
\text { MA: Ros vs. nat }\end{array}$ & 12 weeks & $-65.3 \%$ & unchanged & NA \\
\hline $\begin{array}{l}\text { T2DM } \\
\text { hypertensive }\end{array}$ & $\begin{array}{l}\text { Sarafidis et al. } \\
2005[44]\end{array}$ & observational & 20 & $4 \mathrm{mg}$ Ros & 26 weeks & $-35 \%$ & $-5.4 /-4.1$ & NA \\
\hline DM with ESRD & $\begin{array}{l}\text { Mohideen et al. } \\
2005[45]\end{array}$ & $\begin{array}{l}\text { open label, } \\
\text { randomized, } \\
\text { controlled }\end{array}$ & 12 & $\begin{array}{l}\text { Tro+Base diabetes } \\
\text { medicine vs. base } \\
\text { diabetes medicine }\end{array}$ & 6 months & NA & NA & $\begin{array}{l}\text { safe and effective } \\
\text { hyperglycemia } \\
\text { control }\end{array}$ \\
\hline T2DM & $\begin{array}{l}\text { Bakris et al. } \\
2006[46]\end{array}$ & $\begin{array}{l}\text { double-blind, } \\
\text { parallel-group }\end{array}$ & 389 & Ros vs. Gli & 32 weeks & $-22.7 \%$ & $-3.4 /-2.5$ & NA \\
\hline T2DM & $\begin{array}{l}\text { Miyazaki et al. } \\
2007 \text { [47] }\end{array}$ & $\begin{array}{l}\text { double-blind, } \\
\text { randomized } \\
\text { controlled }\end{array}$ & 29 & Ros vs. placebo & 3 months & $-53.7 \%$ & unchanged & GFR unchanged \\
\hline $\begin{array}{l}\mathrm{DN}(\mathrm{CKD} 3 \text { or } \\
4 \text { stage) }\end{array}$ & $\begin{array}{l}\text { Jin and Pan } \\
2007[48]\end{array}$ & $\begin{array}{l}\text { randomized } \\
\text { controlled }\end{array}$ & 60 & $\begin{array}{l}\text { Pio } 30 \mathrm{mg}+\text { losartan } \\
(100 \mathrm{mg}) \text { vs. losartan } \\
(100 \mathrm{mg})\end{array}$ & 1 year & $\begin{array}{l}\text { decrease } \\
\text { proteinuria }\end{array}$ & NA & $\begin{array}{l}\text { slower decrease } \\
\text { in GFR }\end{array}$ \\
\hline $\begin{array}{l}\text { Non-diabetic } \\
\text { renal disease }\end{array}$ & $\begin{array}{l}\text { Kincaid-Smith } \\
\text { et al., } 2008 \text { [49] }\end{array}$ & $\begin{array}{l}\text { open-label random- } \\
\text { ized controlled cross- } \\
\text { over study }\end{array}$ & 40 & $\begin{array}{l}\text { Ros } 4 \text { mg vs. } \\
\text { usual treatment } \\
\text { (ACEI/ARB) }\end{array}$ & 4 months & $-22.9 \%$ & $-7.8 / \mathrm{NS}$ & NA \\
\hline
\end{tabular}

$\mathrm{BP}=$ Blood pressure; $\mathrm{NA}=$ not available; $\mathrm{MA}=$ microalbuminuria $;$ Tro = trosiglitazone; Pio = pioglitazone; Ros = rosiglitazone; Met = metformin; Gli = glibenclamide; Vog = voglibose; NS = not significant.

${ }^{1}$ The complete reference list for this table is available as online supplementary material (www.karger.com/doi/10.1159/000224789). 
transcription of multiple genes has raised the possible utility of this class of compounds in other diseases beyond diabetes. The major off-target effect of TZDs is fluid retention and this may underlie the recent reports of increased heart failure. This could be managed by patient selection and careful monitoring. However, concerns over the increase in all-cause and cardiovascular mortality with TZDs (especially rosiglitazone) are likely to reduce prescribing of TZDs in patients with risk factors for IHD - these include CKD and DN patients. The recent development of partial agonists to PPAR- $\gamma$ could be one solution to reducing the off-target effects of full agonists
[87]. Further preclinical and clinical studies are needed to establish the true risk-benefit ratio for using TZDs in non-diabetic nephropathies, especially 'orphan diseases' without any effective treatment.

\section{Acknowledgements}

We thank Prof. Changlin Mei for support and encouragement. Z.M. was supported by a Fellowship from the International Society of Nephrology (ISN) and a Young Investigator Award from Changzheng Hospital. A.C.M.O. is a Wellcome Trust Research Leave Senior Fellow.

\section{References}

1 Issemann I, Green S: Activation of a member of the steroid hormone receptor superfamily by peroxisome proliferators. Nature 1990; 347:645-650.

-2 Cattley RC, DeLuca J, Elcombe C, FennerCrisp P, Lake BG, Marsman DS, Pastoor TA, Popp JA, Robinson DE, Schwetz B, Tugwood J, Wahli W: Do peroxisome proliferating compounds pose a hepatocarcinogenic hazard to humans? Regul Toxicol Pharmacol 1998;27:47-60.

-3 Drazen JM, Morrissey S, Curfman GD: Rosiglitazone: continued uncertainty about safety. N Engl J Med 2007;357:63-64.

4 Guan Y, Breyer MD: Peroxisome proliferator-activated receptors (PPARs): novel therapeutic targets in renal disease. Kidney Int 2001;60:14-30.

$\checkmark 5$ Fajas L, Debril MB, Auwerx J: Peroxisome proliferator-activated receptor-gamma: from adipogenesis to carcinogenesis. J Mol Endocrinol 2001;27:1-9.

-6 Desvergne B, Wahli W: Peroxisome proliferator-activated receptors: nuclear control of metabolism. Endocr Rev 1999;20:649-688.

$\checkmark 7$ Kim HJ, Woo IS, Kang ES, Eun SY, Lee JH, Chang KC, Kim JH, Seo HG: Identification of a truncated alternative splicing variant of human PPARgammal that exhibits dominant negative activity. Biochem Biophys Res Commun 2006;347:698-706.

$\checkmark 8$ Lehmann JM, Moore LB, Smith-Oliver TA, Wilkison WO, Willson TM, Kliewer SA: An antidiabetic thiazolidinedione is a high affinity ligand for peroxisome proliferator-activated receptor gamma (PPAR gamma). J Biol Chem 1995;270:12953-12956.

-9 Benigni A, Zoja C, Tomasoni S, Campana M, Corna D, Zanchi C, Gagliardini E, Garofano E, Rottoli D, Ito T, Remuzzi G: Transcriptional regulation of nephrin gene by peroxisome proliferator-activated receptor-gamma agonist: molecular mechanism of the antiproteinuric effect of pioglitazone. J Am Soc Nephrol 2006; 17:1624-1632.
10 Yang HC, Ma LJ, Ma J, Fogo AB: Peroxisome proliferator-activated receptor-gamma agonist is protective in podocyte injury-associated sclerosis. Kidney Int 2006;69:17561764

11 Sarafidis PA: Thiazolidinedione derivatives in diabetes and cardiovascular disease: an update. Fundam Clin Pharmacol 2008;22 247-264

12 Brown JD, Plutzky J: Peroxisome proliferator-activated receptors as transcriptional nodal points and therapeutic targets. Circulation 2007; 115:518-533.

13 Arima S, Kohagura K, Takeuchi K, Taniyama Y, Sugawara A, Ikeda Y, Abe M, Omata K, Ito S: Biphasic vasodilator action of troglitazone on the renal microcirculation. J Am Soc Nephrol 2002;13:342-349.

14 Pistrosch F, Herbrig K, Kindel B, Passauer J, Fischer S, Gross P: Rosiglitazone improves glomerular hyperfiltration, renal endothelial dysfunction, and microalbuminuria of incipient diabetic nephropathy in patients. Diabetes 2005;54:2206-2211.

15 Harte A, McTernan P, Chetty R, Coppack S, Katz J, Smith S, Kumar S: Insulin-mediated upregulation of the renin angiotensin system in human subcutaneous adipocytes is reduced by rosiglitazone. Circulation 2005; 111:1954-1961.

-16 Efrati S, Berman S, Ilgiyeav E, Averbukh Z, Weissgarten J: PPAR-gamma activation inhibits angiotensin II synthesis, apoptosis, and proliferation of mesangial cells from spontaneously hypertensive rats. Nephron Exp Nephrol 2007;106:e107-e112.

17 Schiffrin EL, Amiri F, Benkirane K, Iglarz M, Diep QN: Peroxisome proliferator-activated receptors: vascular and cardiac effects in hypertension. Hypertension 2003;42: 664-668.
18 Omasu F, Oda T, Yamada M, Yoshizawa N, Yamakami K, Sakurai Y, Miura S: Effects of pioglitazone and candesartan on renal fibrosis and the intrarenal plasmin cascade in spontaneously hypercholesterolemic rats. Am J Physiol Renal Physiol 2007;293:F1292F1298.

19 Namikoshi T, Tomita N, Satoh M, Haruna Y, Kobayashi S, Komai N, Sasaki T, Kashihara N: Pioglitazone enhances the antihypertensive and renoprotective effects of candesartan in Zucker obese rats fed a high-protein diet. Hypertens Res 2008;31:745-755.

20 Miyazaki Y, Cersosimo E, Triplitt C, DeFronzo RA: Rosiglitazone decreases albuminuria in type 2 diabetic patients. Kidney Int 2007;72:1367-1373.

-21 Agarwal R: Anti-inflammatory effects of short-term pioglitazone therapy in men with advanced diabetic nephropathy. Am J Physiol Renal Physiol 2006;290:F600-F605.

22 Ko GJ, Kang YS, Han SY, Lee MH, Song HK, Han KH, Kim HK, Han JY, Cha DR: Pioglitazone attenuates diabetic nephropathy through an anti-inflammatory mechanism in type 2 diabetic rats. Nephrol Dial Transplant 2008;23:2750-2760.

23 Efrati S, Berman S, Chachashvili A, Cohen $\mathrm{N}$, Averbukh Z, Weissgarten J: Rosiglitazone treatment attenuates expression of inflammatory hallmarks in the remaining kidney following contralateral nephrectomy. Am J Nephrol 2008;28:238-245.

24 Efrati S, Berman S, Chachashvili A, Cohen N, Siman-Tov Y, Averbukh Z, Weissgarten J: Rosiglitazone treatment attenuates renal tissue inflammation generated by urinary tract obstruction. Nephrology (Carlton) 2009;14: 189-197.

25 Sener G, Sehirli AO, Gedik N, Dülger GA: Rosiglitazone, a PPAR-gamma ligand, protects against burn-induced oxidative injury of remote organs. Burns 2007;33:587-593. 
26 Mohideen P, Bornemann M, Sugihara J, Genadio V, Sugihara V, Arakaki R: The metabolic effects of troglitazone in patients with diabetes and end-stage renal disease. Endocrine 2005;28:181-186.

27 Anderson PW, Zhang XY, Tian J, Correale JD, Xi XP, Yang D, Graf K, Law RE, Hsueh WA: Insulin and angiotensin II are additive in stimulating TGF-beta 1 and matrix mRNAs in mesangial cells. Kidney Int 1996; 50:745-753.

28 Morrisey K, Evans RA, Wakefield L, Phillips AO: Translational regulation of renal proximal tubular epithelial cell transforming growth factor-betal generation by insulin. Am J Pathol 2001;159:1905-1915.

-29 Panchapakesan U, Pollock CA, Chen XM: The effect of high glucose and PPAR-gamma agonists on PPAR-gamma expression and function in HK-2 cells. Am J Physiol Renal Physiol 2004;287:F528-F534.

30 Perico N, Remuzzi G: Inhibition of TGF-beta expression: a novel role for thiazolidinediones to implement renoprotection in diabetes. Kidney Int 2007;72:1419-1421.

- 31 Ohtomo S, Izuhara Y, Takizawa S, Yamada N, Kakuta T, van Ypersele de Strihou C, Miyata $\mathrm{T}$ : Thiazolidinediones provide better renoprotection than insulin in an obese, hypertensive type II diabetic rat model. Kidney Int 2007;72:1512-1519.

- 32 Isshiki K, Haneda M, Koya D, Maeda S, Sugimoto T, Kikkawa R: Thiazolidinedione compounds ameliorate glomerular dysfunction independent of their insulin-sensitizing action in diabetic rats. Diabetes 2000;49: 1022-1032.

-33 Ma LJ, Marcantoni C, Linton MF, Fazio S, Fogo AB: Peroxisome proliferator-activated receptor-gamma agonist troglitazone protects against nondiabetic glomerulosclerosis in rats. Kidney Int 2001;59:1899-1910.

- 34 Kawai T, Masaki T, Doi S, Arakawa T, Yokoyama Y, Doi T, Kohno N, Yorioka N: PPAR-gamma agonist attenuates renal interstitial fibrosis and inflammation through reduction of TGF-beta. Lab Invest 2009;89: $47-58$.

- 35 Dong FQ, Li H, Cai WM, Tao J, Li Q, Ruan Y, Zheng FP, Zhang Z: Effects of pioglitazone on expressions of matrix metalloproteinases 2 and 9 in kidneys of diabetic rats. Chin Med J (Engl) 2004;117:1040-1044.

- 36 Derosa G, Cicero AF, Gaddi A, Ragonesi PD, Piccinni MN, Fogari E, Salvadeo S, Ciccarelli L, Fogari R: A comparison of the effects of pioglitazone and rosiglitazone combined with glimepiride on prothrombotic state in type 2 diabetic patients with the metabolic syndrome. Diabetes Res Clin Pract 2005;69: 5-13.

- 37 Zafiriou S, Stanners SR, Saad S, Polhill TS, Poronnik P, Pollock CA: Pioglitazone inhibits cell growth and reduces matrix production in human kidney fibroblasts. J Am Soc Nephrol 2005;16:638-645.
38 Chatterjee PK: Novel pharmacological approaches to the treatment of renal ischemiareperfusion injury: a comprehensive review. Naunyn Schmiedebergs Arch Pharmacol 2007;376:1-43.

39 Sivarajah A, Chatterjee PK, Patel NS, Todorovic Z, Hattori Y, Brown PA, Stewart KN, Mota-Filipe H, Cuzzocrea S, Thiemermann C: Agonists of peroxisome-proliferator activated receptor-gamma reduce renal ischemia/reperfusion injury. Am J Nephrol 2003; 23:267-276.

40 Doi S, Masaki T, Arakawa T, Takahashi S, Kawai T, Nakashima A, Naito T, Kohno N, Yorioka N: Protective effects of peroxisome proliferator-activated receptor gamma ligand on apoptosis and hepatocyte growth factor induction in renal ischemia-reperfusion injury. Transplantation 2007;84:207213.

-41 Pereira MG, Camara NO, Campaholle G, Cenedeze MA, de Paula Antunes Teixeira V, dos Reis MA, Pacheco-Silva A: Pioglitazone limits cyclosporine nephrotoxicity in rats. Int Immunopharmacol 2006;6:1943-1951.

42 Chung BH, Li C, Sun BK, Lim SW, Ahn KO, Yang JH, Choi YH, Yoon KH, Sugawara A, Ito S, Kim J, Yang CW: Rosiglitazone protects against cyclosporine-induced pancreatic and renal injury in rats. Am J Transplant 2005;5:1856-1867.

43 Muto S, Aiba A, Saito Y, Nakao K, Nakamura K, Tomita K, Kitamura T, Kurabayashi M, Nagai R, Higashihara E, Harris PC, Katsuki M, Horie S: Pioglitazone improves the phenotype and molecular defects of a targeted pkd1 mutant. Hum Mol Genet 2002;11:17311742.

44 Meirhaeghe A, Fajas L, Helbecque N, Cottel D, Lebel P, Dallongeville J, Deeb S, Auwerx J, Amouyel P: A genetic polymorphism of the peroxisome proliferator-activated receptor gamma gene influences plasma leptin levels in obese humans. Hum Mol Genet 1998;7: 435-440.

45 Orio F Jr, Matarese G, Di Biase S, Palomba S, Labella D, Sanna V, Savastano S, Zullo F, Colao A, Lombardi G: Exon 6 and 2 peroxisome proliferator-activated receptor-gamma polymorphisms in polycystic ovary syndrome. J Clin Endocrinol Metab 2003;88:5887-5892.

46 Pollex RL, Mamakeesick M, Zinman B, Harris SB, Hegele RA, Hanley AJ: Peroxisome proliferator-activated receptor gamma polymorphism prol2ala is associated with nephropathy in type 2 diabetes. J Diabetes Complications 2007;21:166-171.

47 Jorsal A, Tarnow L, Lajer M, Ek J, Hansen T, Pedersen O, Parving HH: The PPAR gamma 2 Pro12Ala variant predicts ESRD and mortality in patients with type 1 diabetes and diabetic nephropathy. Mol Genet Metab 2008;94:347-351.

48 Meirhaeghe A, Amouyel P: Impact of genetic variation of PPARgamma in humans. Mol Genet Metab 2004;83:93-102.
49 Schernthaner G, Matthews DR, Charbonnel B, Hanefeld M, Brunetti P: Efficacy and safety of pioglitazone versus metformin in patients with type 2 diabetes mellitus: a doubleblind, randomized trial. J Clin Endocrinol Metab 2004;89:6068-6076.

50 Imano E, Kanda T, Nakatani Y, Nishida T, Arai K, Motomura M, Kajimoto Y, Yamasaki Y, Hori M: Effect of troglitazone on microalbuminuria in patients with incipient diabetic nephropathy. Diabetes Care 1998;21:21352139.

51 Nakamura T, Ushiyama C, Shimada N, Hayashi K, Ebihara I, Koide H: Comparative effects of pioglitazone, glibenclamide, and voglibose on urinary endothelin-1 and albumin excretion in diabetes patients. J Diabetes Complications 2000;14:250-254.

52 Nakamura T, Ushiyama C, Suzuki S, Shimada N, Sekizuka K, Ebihara L, Koide H: Effect of troglitazone on urinary albumin excretion and serum type IV collagen concentrations in type 2 diabetic patients with microalbuminuria or macroalbuminuria. Diabet Med 2001;18:308-313.

53 Hanefeld M, Brunetti P, Schernthaner GH, Matthews DR, Charbonnel BH: One-year glycemic control with a sulfonylurea plus pioglitazone versus a sulfonylurea plus metformin in patients with type 2 diabetes. Diabetes Care 2004;27:141-147.

54 Bakris GL, Ruilope LM, McMorn SO, Weston WM, Heise MA, Freed MI, Porter LE: Rosiglitazone reduces microalbuminuria and blood pressure independently of glycemia in type 2 diabetes patients with microalbuminuria. J Hypertens 2006;24:2047-2055.

55 Bakris G, Viberti G, Weston WM, Heise M, Porter LE, Freed MI: Rosiglitazone reduces urinary albumin excretion in type II diabetes. J Hum Hypertens 2003;17:7-12.

56 Sarafidis PA, Lasaridis AN, Nilsson PM, Hitoglou-Makedou AD, Pagkalos EM, Yovos JG, Pliakos CI, Tourkantonis AA: The effect of rosiglitazone on urine albumin excretion in patients with type 2 diabetes mellitus and hypertension. Am J Hypertens 2005;18:227234

57 Jin HM, Pan Y: Renoprotection provided by losartan in combination with pioglitazone is superior to renoprotection provided by losartan alone in patients with type 2 diabetic nephropathy. Kidney Blood Press Res 2007;30:203-211.

58 Kincaid-Smith P, Fairley KF, Farish S, Best JD, Proietto J: Reduction of proteinuria by rosiglitazone in non-diabetic renal disease. Nephrology (Carlton) 2008;13:58-62.

59 Joy MS, Gipson DS, Dike M, Powell L, Thompson A, Vento S, Eddy A, Fogo AB, Kopp JB, Cattran D, Trachtman H: Phase I trial of rosiglitazone in FSGS: I. Report of the FONT Study Group. Clin J Am Soc Nephrol 2009;4:39-47. 
-60 Budde K, Neumayer HH, Fritsche L, Sulowicz W, Stompor T, Eckland D: The pharmacokinetics of pioglitazone in patients with impaired renal function. Br J Clin Pharmacol 2003;55:368-374.

-61 Nissen SE, Wolski K: Effect of rosiglitazone on the risk of myocardial infarction and death from cardiovascular causes. N Engl J Med 2007;356:2457-2471.

62 Ohata M, Suzuki H, Sakamoto K, Hashimoto K, Nakajima H, Yamauchi M, Hokkyo K, Yamada H, Toda G: Pioglitazone prevents acute liver injury induced by ethanol and lipopolysaccharide through the suppression of tumor necrosis factor-alpha. Alcohol Clin Exp Res 2004;28:139S-144S.

-63 Neuschwander-Tetri BA, Brunt EM, Wehmeier KR, Oliver D, Bacon BR: Improved nonalcoholic steatohepatitis after 48 weeks of treatment with the PPAR-gamma ligand rosiglitazone. Hepatology 2003;38:10081017.

64 Lutchman G, Modi A, Kleiner DE, Promrat K, Heller T, Ghany M, Borg B, Loomba R, Liang TJ, Premkumar A, Hoofnagle JH: The effects of discontinuing pioglitazone in patients with nonalcoholic steatohepatitis. Hepatology 2007;46:424-429.

-65 Giral H, Villa-Bellosta R, Catalan J, Sorribas $\mathrm{V}$ : Cytotoxicity of peroxisome proliferatoractivated receptor alpha and gamma agonists in renal proximal tubular cell lines. Toxicol In Vitro 2007;21:1066-1076.

66 Kwon CH, Yoon CS, Kim YK: Ciglitazone induces caspase-independent apoptosis via p38-dependent AIF nuclear translocation in renal epithelial cells. Toxicology 2008;244: 13-24.

67 Michalik L, Desvergne B, Wahli W: Peroxisome-proliferator-activated receptors and cancers: complex stories. Nat Rev Cancer 2004;4:61-70.

-68 Tiwari S, Blasi ER, Heyen JR, McHarg AD, Ecelbarger CM: Time course of AQP-2 and $\mathrm{ENaC}$ regulation in the kidney in response to PPAR agonists associated with marked edema in rats. Pharmacol Res 2008;57:383-392.

-69 Guan Y, Hao C, Cha DR, Rao R, Lu W, Kohan DE, Magnuson MA, Redha R, Zhang Y, Breyer MD: Thiazolidinediones expand body fluid volume through PPARgamma stimulation of ENaC-mediated renal salt absorption. Nat Med 2005; 11:861-866.
70 Rennings AJ, Smits P, Stewart MW, Tack CJ: Fluid retention and vascular effects of rosiglitazone in obese, insulin-resistant, nondiabetic subjects. Diabetes Care 2006;29:581587.

71 Walker AB, Naderali EK, Chattington PD, Buckingham RE, Williams G: Differential vasoactive effects of the insulin sensitizers rosiglitazone (BRL 49653) and troglitazone on human small arteries in vitro. Diabetes 1998;47:810-814.

72 Basu A, Jensen MD, McCann F, Mukhopadhyay D, Joyner MJ, Rizza RA: Effects of pioglitazone versus glipizide on body fat distribution, body water content, and hemodynamics in type 2 diabetes. Diabetes Care 2006;29:510-514.

73 Sarafidis PA, Lasaridis AN, Nilsson PM, Mouslech TF, Hitoglou-Makedou AD, Stafylas PC, Kazakos KA, Yovos JG, Tourkantonis AA: The effect of rosiglitazone on novel atherosclerotic risk factors in patients with type 2 diabetes mellitus and hypertension: an open-label observational study. Metabolism 2005;54:1236-1242.

74 Lebovitz HE: Differentiating members of the thiazolidinedione class: a focus on safety. Diabetes Metab Res Rev 2002;18(suppl 2): S23-S29.

75 Dormandy JA, Charbonnel B, Eckland DJ, Erdmann E, Massi-Benedetti M, Moules IK, Skene AM, Tan MH, Lefebvre PJ, Murray GD, Standl E, Wilcox RG, Wilhelmsen L, Betteridge J, Birkeland K, Golay A, Heine RJ, Koranyi L, Laakso M, Mokan M, Norkus A, Pirags V, Podar T, Scheen A, Scherbaum W, Schernthaner G, Schmitz O, Skrha J, Smith U, Taton J: Secondary prevention of macrovascular events in patients with type 2 diabetes in the proactive study (prospective pioglitazone clinical trial in macrovascular events): a randomised controlled trial. Lancet 2005;366:1279-1289.

76 Patel C, Wyne KL, McGuire DK: Thiazolidinediones, peripheral oedema and congestive heart failure: what is the evidence? Diab Vasc Dis Res 2005;2:61-66.

77 Mersmann J, Tran N, Zacharowski PA, Grotemeyer D, Zacharowski K: Rosiglitazone is cardioprotective in a murine model of myocardial I/R. Shock 2008;30:64-68.

78 Yue TL, Bao W, Gu JL, Cui J, Tao L, Ma XL, Ohlstein EH, Jucker BM: Rosiglitazone treatment in Zucker diabetic Fatty rats is associated with ameliorated cardiac insulin resistance and protection from ischemia/reperfusion-induced myocardial injury. Diabetes 2005;54:554-562.
79 Molavi B, Chen J, Mehta JL: Cardioprotective effects of rosiglitazone are associated with selective overexpression of type 2 angiotensin receptors and inhibition of $\mathrm{p} 42 / 44$ MAPK. Am J Physiol Heart Circ Physiol 2006;291:H687-H693.

80 Lago RM, Singh PP, Nesto RW: Congestive heart failure and cardiovascular death in patients with prediabetes and type 2 diabetes given thiazolidinediones: a meta-analysis of randomised clinical trials. Lancet 2007;370: 1129-1136.

81 Home PD, Pocock SJ, Beck-Nielsen H, Gomis R, Hanefeld M, Jones NP, Komajda M, McMurray JJ: Rosiglitazone evaluated for cardiovascular outcomes - an interim analysis. N Engl J Med 2007;357:28-38.

82 Diamond GA, Bax L, Kaul S: Uncertain effects of rosiglitazone on the risk for myocardial infarction and cardiovascular death. Ann Intern Med 2007; 147:578-581.

83 Schneider CA, Ferrannini E, Defronzo R, Schernthaner G, Yates J, Erdmann E: Effect of pioglitazone on cardiovascular outcome in diabetes and chronic kidney disease. J Am Soc Nephrol 2008;19:182-187.

84 Chiquette E, Ramirez G, Defronzo R: A meta-analysis comparing the effect of thiazolidinediones on cardiovascular risk factors. Arch Intern Med 2004;164:2097-2104.

85 Stafylas PC, Sarafidis PA, Lasaridis AN: The controversial effects of thiazolidinediones on cardiovascular morbidity and mortality. Int J Cardiol 2009;131:298-304.

$>86$ Nesto RW, Bell D, Bonow RO, Fonseca V, Grundy SM, Horton ES, Le Winter M, Porte D, Semenkovich CF, Smith S, Young LH, Kahn R: Thiazolidinedione use, fluid retention, and congestive heart failure: a consensus statement from the American Heart Association and American Diabetes Association. Diabetes Care 2004;27:256-263.

$>87$ Artis DR, Lin JJ, Zhang C, Wang W, Mehra U, Perreault M, Erbe D, Krupka HI, England BP, Arnold J, Plotnikov AN, Marimuthu A, Nguyen H, Will S, Signaevsky M, Kral J, Cantwell J, Settachatgull C, Yan DS, Fong D, Oh A, Shi S, Womack P, Powell B, Habets G, West BL, Zhang KY, Milburn MV, Vlasuk GP, Hirth KP, Nolop K, Bollag G, Ibrahim PN, Tobin JF: Scaffold-based discovery of indeglitazar, a PPAR pan-active anti-diabetic agent. Proc Natl Acad Sci USA 2009;106: 262-267. 


\section{Editorial Comment}

M. El Nahas, Sheffield

The comprehensive review by Mao and Ong in this issue of Nephron Clinical Practice reminds the reader that PPAR- $\gamma$ agonists have attracted great attention in recent years due to their roles in the regulation of key metabolic, inflammatory, atherosclerotic pathways and in kidney protection. As a consequence, thiazolidinediones (TZDs), the most widely clinically used PPAR- $\gamma$ agonists, have become, over the last decade, blockbuster drugs in the management of type 2 diabetes mellitus (T2DM). These agents promised a lot, delivered a little and are now struggling to maintain a role in the management of diabetes in view of a high risk/benefit ratio. As highlighted in the review, they promised improved metabolic control, reduction and slowing of the progression of diabetic microvascular complications including diabetic nephropathy. They did indeed prove effective in reducing proteinuria in diabetic and even some non-diabetic chronic kidney diseases (CKD), along with a reduction in systemic blood pressure. But the TZDs are associated with significant fluid retention and have been shown to exacerbate congestive heart failure. Controversy remains as to whether they are associated with increased all-cause and/or cardiovascular mortality. In elderly patients with T2DM, CKD is often associated with diffuse atherosclerotic vascular disease. These agents should be used with extreme caution, if at all, in these individuals. Furthermore, recent data suggest that rosiglitazone is also associated with increased mortality in diabetic patients on haemodialysis [Ramirez et al.: J Am Soc Nephrol 2009 Apr 8, Epub ahead of print]. Patients with CKD, in general, are at the highest risk of cardiovascular morbidity and mortality, the use of potentially cardiotoxic agents should be contraindicated. Mao and Ong draw attention to the development of partial agonists of PPAR- $\gamma$ that may have a more acceptable profile. They conclude that such agents may still have a role to play in 'orphan kidney diseases' without any effective treatment. Only time will tell whether TZDs have a future... 